Archives de sciences sociales des religions

176 | octobre-décembre 2016

Bulletin Bibliographique

\title{
Astrid Von Busekist, Portes et murs. Des frontières en démocratie
}

Paris, Albin Michel, 2016, 224 p.

Madalina Vârtejanu-Joubert

\section{CpenEdition}

\section{Journals}

Édition électronique

URL : http://journals.openedition.org/assr/28197

DOI : $10.4000 /$ assr.28197

ISSN : $1777-5825$

Éditeur

Éditions de l'EHESS

Édition imprimée

Date de publication : 31 décembre 2016

Pagination : 282

ISSN : 0335-5985

Référence électronique

Madalina Vârtejanu-Joubert, " Astrid Von Busekist, Portes et murs. Des frontières en démocratie », Archives de sciences sociales des religions [En ligne], 176 | octobre-décembre 2016, mis en ligne le 17 juillet 2017, consulté le 23 septembre 2020. URL : http://journals.openedition.org/assr/28197; DOI https://doi.org/10.4000/assr.28197

Ce document a été généré automatiquement le 23 septembre 2020.

(C) Archives de sciences sociales des religions 


\section{Astrid Von Busekist, Portes et murs. Des frontières en démocratie}

Paris, Albin Michel, 2016, 224 p.

Madalina Vârtejanu-Joubert

\section{RÉFÉRENCE}

Astrid von Busekist, Portes et murs. Des frontières en démocratie, Paris, Albin Michel, 2016, $224 \mathrm{p}$.

1 L'ouvrage dont nous rendons compte ici pourrait passer inaperçu dans le domaine des sciences des religions ou des études juives puisque son titre ne laisse pas deviner une telle inscription académique. Il est certes question de "portes et murs » ainsi que de «frontières en démocratie ", mais, de fait, c'est le dossier de l'eruv dans le monde occidental contemporain qui constitue le point de départ et fournit à l'ouvrage sa matière première. La loi juive interdit de se déplacer le jour de shabbat à l'extérieur de l'espace privé, mais elle conçoit également une soupape symbolique : mélanger espace privé et espace public en l'entourant d'une clôture provisoire, souvent invisible et formée par des éléments existants, et constituant ainsi un eruv. L'intérêt du livre est multiple : tout d'abord, il fait œuvre interdisciplinaire, car il aborde, par le prisme de la science politique, l'un des objets habituellement traités dans le cadre des disciplines de l'érudition. Ensuite, il ouvre un débat plus large sur la place et le rôle de la religion dans la démocratie et dans les sociétés libérales. Enfin, il met en lumière l'enchevêtrement contemporain des régimes d'historicités et les mécanismes d'actualisation du passé en prenant comme exemple un certain pan du judaïsme contemporain. Réalité marginale de nos sociétés occidentales, l'auteur retient l'eruv comme un miroir grossissant des rapports entre communauté et société, entre majorité et minorité, entre religion et État.

2 L'auteur part de l'hypothèse que, du moins en théorie, il existe une incompatibilité de fond entre la démocratie libérale et l'idée de frontière (p. 41 et p.132). Elle fait 
également le constat que l'eruv, frontière temporaire et invisible destinée au mélange et non à la séparation, est là pour rappeler le caractère relatif de ce postulat (p. 61). À cela s'ajoute un troisième fait: l'opposition que l'installation d'un eruv a pu susciter dans certaines villes, notamment aux États-Unis, au Canada et en Australie, mais aussi en Israël et en Angleterre. Ainsi, l'auteur se demande de quelle rationalité cette dissonance cognitive tient-elle. Au sein d'une apparente contradiction théorique et d'une réelle conflictualité sur le terrain, quels dispositifs peut-on mobiliser pour comprendre les effets de l'eruv dans les démocraties libérales de type occidental ?

Von Busekist raisonne avant tout en théoricienne de la science politique. Elle construit son argumentation en partant de travaux théoriques qui servent, ensuite, à expliquer des situations concrètes où la construction d'un eruv a donné lieu à des polémiques allant parfois jusqu'au procès en justice: Outremont (Québec, Canada), Beachwood (Ohio), Belle Harbor (New York), Long Branch et Tenafly (New Jersey), Kyrias Joel (New York), Barnet, Angleterre. Elle fait remarquer que, tout comme la frontière, le conflit ne fait pas que séparer, mais contribue à la recherche et la construction de ponts, matérialisés dans ce cas dans le processus de traduction d'une logique religieuse vers un langage laïque et libéral. L'opération de traduction est imposée, selon l'auteur, par la coexistence et la compétition de deux univers normatifs, impérial et païdéique, termes repris du dispositif théorique de Robert Cover. Le premier est un corpus alors que le deuxième est une signification; le premier est universaliste, le deuxième particulariste ; le premier faible dans son principe, le deuxième puissant. La description de ces logiques de traduction destinées à négocier la coexistence représente le principal apport du livre. Le principe de la traduction est décrit à trois niveaux : la justification de l'eruv au sein des diverses communautés juives qui réclament son installation, la justification des principes de tolérance au sein de la majorité du lieu, l'argumentation du juge appelé à prendre parti dans ce conflit normatif.

4 Ainsi, trois possibles logiques président à la justification de l'eruv vis-à-vis du monde extérieur à la communauté pratiquante: hétérologique (« Ma religion me le demande. J'obéis aux commandements, je n'ai de compte à rendre à personne ») ; logique (« Pour que vous compreniez la manière dont j'agis, je vous explique la logique à l'œuvre. Il y a une rationalité interne que vous pouvez entendre et reconnaitre»); homologique ( « justifie dans le vocabulaire et depuis la logique de la raison publique, pourquoi il est important, pour des raisons acceptables par - et compatibles avec-le droit séculier, que ma demande soit entendue »). Pour exemple, l'eruv peut être considéré comme légitime puisqu'il assure " la libre circulation dans, et le libre accès à l'espace public, l'égalité entre les citoyens et entre les sexes» (p.81) et puisqu'il " est un moyen de sociabilité de la communauté à la fois interne et externe - il force les murs de la maison et permet à tous d'accéder, en famille, à l'espace public » (p. 90).

En face, le nomos impérial de type libéral, n'est pas toujours hostile et trouve des justifications à l'installation d'un eruv dans les modèles de l'affirmative action selon laquelle "les exemptions qui privilégient volontairement des minorités (au lieu de peser involontairement sur les minorités) sont, sous ces conditions, justifiées, parce que nous n'avons pas à nous interroger, en tant que libéraux non sceptiques, sur le contenu substantiel des croyances de chacun. Il n'y a aucune raison à ce que les motifs religieux aient une préséance sur d'autres motifs, mais il n'y a non plus aucune raison pour qu'ils soient invalidés parce qu'ils ont un caractère religieux » (p. 129). L'auteur illustre ces principes par quelques arguments positifs invoqués: le caractère 
œcuménique d'une ville, le principe de solidarité, l'eruv comme outil au service de "l'intérêt public » de la minorité religieuse ou comme une contribution au bien public (p. 130-131).

6 Mais qu'est-ce qui fait que, dans certains cas, c'est l'opposition plus ou moins généralisée d'un quartier qui se manifeste? La réponse de l'auteur passe par la sémiotique de l'espace : « tout se passe comme si l'espace normatif majoritaire, celui de la nation, de ses rites et de ses symboles, de son empreinte chrétienne aussi, était "défiguré" par l'impact de signes non majoritaires qui brouillent l'identité historique des lieux et interdisent toute lecture univoque. » (p. 156). Ou encore : « De l'État-nation au quartier, les citoyens ont la même lecture de l'espace.» (p. 157) Dans un tel univers mental, il faut interroger le sens du rite : s'il est signifiant pour les juifs orthodoxes, il est vide pour les autres ou il n'a pas d'autre signifié que celui que les autres lui prêtent. Enjeu de traduction encore, et d'incompréhension, le rite " exprime pour le Moderne, une pensée englobante qui ignore les frontières du privé. Et pour celui, très peu moderne, qui ne connaît que sa culture et sa religion, il perturbe l'ordre immuable du temps. » (p. 173). «Ouverture » pour les Juifs pratiquants, «mur » pour les autres : tel est le conflit des interprétations qui se noue autour de l'eruv.

$7 \quad$ Lorsque ce conflit est porté devant les tribunaux, c'est le juge qui est appelé à trancher au sein de ce pluralisme interprétatif. L'exercice est des plus délicats puisqu'il s'agit d'intervenir dans la logique interne d'une religion, dans le cas d'espèce de juger si le commandement de l'eruv est essentiel ou s'il tient de la commodité, s'il est une expression ou une pratique religieuse. L'arbitrage est d'autant plus difficile que l'eruv est une frontière imaginaire, dématérialisée la plupart du temps et, donc, peu ou pas intrusive. L'auteur conclut en attirant notre attention sur le fait que toute décision prend en compte l'individu en tant que sujet de droit et non pas la communauté dont l' eruv est l'émanation.

8 Après avoir interrogé l'eruv en tant que révélateur de la négociation des frontières symboliques au sein de la démocratie libérale, l'auteur se penche, en conclusion, sur l' eruv en tant que révélateur d'une théorie politique juive. Ainsi, le régime d'historicité du passé met à mal celui du présent. L'antique acception de l'eruv en tant que garant de la commensalité fait surface en constituant le socle de la pérennité du rite. En faisant sienne l'analyse très serrée des textes rabbiniques effectuée par Charlotte Elisheva Fonrobert, l'auteur révèle également le rôle joué par l'eruv dans l'inscription de la communauté orthodoxe au sein d'un espace non-juif ou diasporique. Dans cette configuration, le juif a besoin de l'accord du gentil pour que l'espace public soit considéré durant une journée comme un espace privé : l'étranger est ainsi intégré dans le système halachique et, dit l'auteur, est opéré un « universalisme par le bas » (p. 73).

9 Il demeure néanmoins un aspect que l'auteur n'aborde pas: celui de l'ampleur du phénomène et du caractère représentatif des cas étudiés. Si les controverses juridiques demeurent marginales, comment cela s'intègre-t-il dans un dispositif explicatif qui mobilise à un haut degré la théorie politique ? La généralité des affirmations au sujet de la modernité, de la tolérance, de la discrimination positive ne contrevient-elle pas au caractère localisé de la polémique située notamment sur le continent nord-américain ? Une analyse de la frontière étatique aurait sans doute contribué à mettre en lumière la particularité de chaque situation. Même si nous reconnaissons l'intérêt de placer dans une perspective générale de la théorie politique un fait communautaire, les données de terrain sont trop souvent invoquées en second lieu, comme précipitées de la théorie, et 
l'approche déductive appliquée de façon trop systématique. Enfin, l'emploi de certaines désignations semble ambigu (juifs rabbiniques, juifs hassidiques, haredim, indigènes, pratique indigène); une définition sociologique et doctrinale plus serrée aurait été bienvenue. 